Bull. Austral. Math. Soc.

$11 \mathrm{~A} 25,05 \mathrm{~A} 10,11 \mathrm{M} 41,11 \mathrm{~B} 75$

VoL. 73 (2006) [79-88]

\title{
ON GENERALISED MÖBIUS INVERSION FORMULAS
}

\author{
Tian-Xiao He, Leetsch C. Hsu and Peter J.S. Shiue
}

We provide a wide class of Möbius inversion formulas in terms of the generalised Möbius functions and their application to the setting of the Selberg multiplicative functions.

\section{INTRODUCTION}

This paper is concerned with the problem of construction for a general type of Möbius inversion formulas for the set of the generalised Möbius function $\mu_{\alpha}$. Other types of generalised Möbius inversion formulas can be seen in the recent article by Sándor and Bege [7]. In addition, an application of the Möbius inversion formulas in physics can be found in $[\mathbf{3}]$.

For any $z \in \mathbb{C}$, a generalised Fleck-type Möbius function (see [4]) is defined by

$$
\mu_{z}(t):=\Pi_{p}(-1)^{e_{p}([|t|])}\left(\begin{array}{c}
z \\
e_{p}([|t|])
\end{array}\right)
$$

for any $t \in \mathbb{C}$, where $[|t|]$ is the integer part of $|t|, p$ runs through all the prime divisors of $[|t|]$, and $e_{p}([|t|])=\operatorname{ord} d_{p}([|t|])$ denotes the highest power $k$ of $p$ such that $p^{k}$ divides $[|t|]$. Obviously, $\mu_{1}(n)=\mu(n), n \in \mathbb{N}$, is the classical Möbius function.

Brown, Hsu, Wang and Shiue in [1] show that $\mu_{z}(z \in \mathbb{C})$ is a multiplicative function, that is, if $m$ and $n$ are relatively prime, then $\mu_{z}(m n)=\mu_{z}(m) \mu_{z}(n)$. In addition, $\mu_{0}(n)$ $=\delta_{1 n}(n \in \mathbb{N})$, the Kronecker symbol, that is, $\mu_{0}(n)$ equals to 1 if $n=1$ and 0 otherwise. It can be easily proved that, for each complex number $z, \mu_{z}$ is not a completely multiplicative function except for $\mu_{0}$, which is completely multiplicative.

Let $E$ denote an arbitrary arithmetical semigroup. The set of all complex-valued arithmetical functions on $E$ will be denoted by $A(E)$. Note that the definition of an arithmetical semigroup $E$ implies that $E$ is countable. For the sake of definiteness, one could if desired express $E$ in the form $E=\left\{a_{1}=1, a_{2}, a_{3}, \ldots\right\}$, where $\left|a_{i}\right| \leqslant\left|a_{i+1}\right|$.

\section{Received 21st September, 2005}

The first author would like to thank IWU for a sabbatical leave during which the research in this paper was carried out. The third author would like to thank UNLV for a sabbatical leave during which the research in this paper was carried out.

Copyright Clearance Centre, Inc. Serial-fee code: 0004-9727/06 \$A2.00+0.00. 
Let $h \in A(E)$ be a nonzero completely multiplicative function. We now give generalised Möbius inversion formulas for the set $M_{h}=\left\{\mu_{z, h}=\mu_{z} h: z \in \mathbb{C}\right\}$ with respect to the the generalised Dirichlet convolution * defined as follows.

DEFINITION 1.1: Let $h \in A(E)$ be a nonzero completely multiplicative function. Given two functions $f, g \in A(E)$, the generalised Dirichlet convolution, denoted by $(f * g)_{h}$, is also in $A(E)$. It is defined by $(f * g)_{h}=(f h) *(g h)$, where $*$ is the Dirichlet convolution; that is,

$$
\begin{aligned}
(f * g)_{h}(n): & =((f h) *(g h))(n) \\
& =\sum_{d \mid n}(f h)(d)(g h)\left(\frac{n}{d}\right) \\
& =\sum_{d \mid n}(f h)\left(\frac{n}{d}\right)(g h)(d)
\end{aligned}
$$

for $n=[|t|]$ with $t \in E$.

Next we recall a fairly more general notation of multiplicativity, introduced first by Selberg [8], which apparently did not prevail in the literature.

DEFinition 1.2: A number-theoretical function $F$ is said to be Selberg-multiplicative if, for each prime $p$, there exists $f_{p}: \mathbb{N}_{0} \mapsto \mathbb{C}$ with $f_{p}(0)=1$ for all but finitely many $p$ such that

$$
F(n)=\Pi_{p} f_{p}\left(e_{p}(n)\right)
$$

holds for every $n \in \mathbb{N}$. The class of all Selberg-multiplicative functions is denoted by $\mathcal{G}$.

DEFInITION 1.3: A Dirichlet formal series $\hat{f}(s)(s \in \mathbb{C})$ of the sequence $\{F(n)\}$ is defined by

$$
\widehat{f}(s)=\sum_{n=1}^{\infty} F(n) n^{-s} \quad(s \neq 0) .
$$

One of the main advantages of this more general notation of multiplicativity is that it can be used without change to define multiplicative functions of several variables.

In next section, we shall show that $\left(M_{h}, *\right)$ is a group with the identity element $\mu_{0, h}=\mu_{0} h\left(\mu_{0}(n)=\delta_{n 1}\right)$ and establish a kind of Möbius type inversion. We shall also apply these results to the setting of Selberg-multiplicative functions class $\left(\mathcal{G}_{h}, *\right)=\{F h$ : $F \in \mathcal{G}\}$ in Section 3 , where $h \in A(E)$ is a nonzero completely multiplicative function with $h(0)=1$. Hence, $h(n)=\Pi_{p} h_{p}\left(e_{p}(n)\right)$ with $h_{p}\left(e_{p}(n)\right):=h\left(p^{e_{p}(n)}\right)$ and $h_{p}(0)=1$. The application is based on the following lemmas.

LEMMA 1.4. Let $\mathcal{G}$ be the Selberg class of multiplicative functions defined in Definition 1.2. If $F \in \mathcal{G}$, then for $s \in \mathbb{C}$ we have the fomal identity

$$
\sum_{n=1}^{\infty} F(n) n^{-s} \equiv \sum_{n=1}^{\infty} n^{-s} \Pi_{p} f_{p}\left(e_{p}(n)\right)=\Pi_{p} \sum_{r=0}^{\infty} f_{p}(r) p^{-r s}
$$


in which the product on the rightmost extends over all prime numbers.

Proof: We can denote $n=\Pi_{p} p^{e_{p}(n)}$. Clearly we have

$$
\text { left hand side of }(1.4)=\sum_{n=1}^{\infty} \frac{\Pi_{p} f_{p}\left(e_{p}(n)\right)}{\Pi_{p} p^{s e_{p}(n)}}=\sum_{n=1}^{\infty} \Pi_{p} \frac{f_{p}\left(e_{p}(n)\right)}{p^{s e_{p}(n)}}
$$

and

$$
\text { right hand side of }(1.4)=\Pi_{p}\left\{\frac{f_{p}(0)}{p^{0 \cdot s}}+\frac{f_{p}(1)}{p^{1 \cdot s}}+\frac{f_{p}(2)}{p^{2 \cdot s}}+\frac{f_{p}(3)}{p^{3 \cdot s}}+\cdots\right\} \text {. }
$$

In addition, on the left hand side of (1.4), for every $n=p_{1}^{r_{1}} p_{2}^{r_{2}} p_{3}^{r_{3}} \cdots$, where $r_{j} \geqslant 0$ and $2 \leqslant$ $p_{1}$

$<p_{2}<\cdots$ are primes, the term

$$
\frac{f_{p_{1}}\left(r_{1}\right) f_{p_{2}}\left(r_{2}\right) f_{p_{3}}\left(r_{3}\right) \cdots}{p_{1}^{s r_{1}} p_{2}^{s r_{2}} p_{3}^{s+3} \cdots}
$$

occurs exactly once in the expansion of the right hand side of of (1.4).

On the other hand, in the right hand side expansion, every term with the form (1.5), corresponds to a term with $n=p_{1}^{r_{1}} p_{2}^{r_{2}} p_{3}^{r_{3}} \cdots$. Hence, the lemma holds.

Lemma 1.4 has its own importance, which can be seen from its special case shown as in [9, Theorem 2.6.1].

From Definition 1.3 and product of series, we immediately have the following lemma.

LEMMA 1.5. Given formal series

$$
\widehat{f}(s)=\sum_{n=1}^{\infty} F(n) n^{-s} \quad \text { and } \quad \hat{g}(s)=\sum_{n=1}^{\infty} G(n) n^{-s}(s \neq 0) .
$$

Then $\widehat{f}(s) \cdot \widehat{g}(s)$ generates the sequence $\left\{\sum_{d \mid n} F(n / d) G(d)\right\}$.

\section{A Generalised Möbius Inversion Formula}

TheOREM 2.1. The generalised Dirichlet convolution is commutative and satisfies the associative law. In addition, $(f * g)_{h}$ is also a multiplicative function whenever $f$ and $g$ are multiplicative functions.

Proof: Obviously, the generalised Dirichlet convolution is commutative. The associativity can be established from the following statement.

$$
\begin{aligned}
\left((f * g)_{h} * u\right)_{h}(n) & =\sum_{d \mid n}(f * g)_{h}(d)(u h)\left(\frac{n}{d}\right) \\
& =\sum_{d \mid n} \sum_{c \mid d}(f h)(c)(g h)\left(\frac{d}{c}\right)(u h)\left(\frac{n}{d}\right)
\end{aligned}
$$




$$
\begin{aligned}
& =\sum_{c \mid n} \sum_{\ell \mid(n / c)}(f h)(c)(g h)(\ell)(u h)\left(\frac{n}{c \ell}\right)\left(\ell=\frac{d}{c}\right) \\
& =\sum_{c \mid n}(f h)(c) \sum_{\ell \mid \frac{n}{c}}(g h)(\ell)(u h)\left(\frac{n}{c \ell}\right) \\
& =\sum_{c \mid n}(f h)(c)(g * u)_{h}\left(\frac{n}{c}\right) \\
& =\left(f *(g * u)_{h}\right)_{h}(n) .
\end{aligned}
$$

We now prove that $(f * g)_{h}$ is also multiplicative whenever $f$ and $g$ are multiplicative functions. If $(a, b)=1$, then

$$
(f * g)_{h}(a b)=\sum_{d \mid a b}(f h)(d)(g h)\left(\frac{a b}{d}\right) .
$$

Let $u=(a, d)$ and $v=(b, d)$. Then $u v=d,(u, v)=1$, and

$$
\begin{aligned}
(f * g)_{h}(a b) & =\sum_{u \mid a} \sum_{v \mid b}(f h)(u v)(g h)\left(\frac{a b}{u v}\right) \\
& =\sum_{u \mid a}(f h)(u)(g h)\left(\frac{a}{u}\right) \sum_{v \mid b}(f h)(v)(g h)\left(\frac{b}{v}\right) \\
& =(f * g)_{h}(a)(f * g)_{h}(b) .
\end{aligned}
$$

This completes the proof of the theorem.

TheOREM 2.2. Let $h \in A(E)$ be a nonzero completely multiplicative function, and let * be the Dirichlet convolution. Then $\left(M_{h}, *\right)$ is a group with the identity element $\mu_{0, h}=\mu_{0} h$.

ProOF: First, the set $M_{h}$ is closed with respect to *: for all $\mu_{\alpha, h}, \mu_{\beta, h} \in M_{h}$,

$$
\begin{aligned}
\left(\mu_{\alpha, h} * \mu_{\beta, h}\right)(n) & =\sum_{d \mid n} \mu_{\alpha}(d) h(d) \mu_{\beta}\left(\frac{n}{d}\right) h\left(\frac{n}{d}\right) \\
& =h(n) \sum_{d \mid n} \mu_{\alpha}(d) \mu_{\beta}\left(\frac{n}{d}\right) \\
& =\mu_{\alpha+\beta, h}(n),
\end{aligned}
$$

where the last step is due to [1, Lemma 2].

Secondly, the convolution * is commutative and associative in $M_{h}$ from Theorem 2.1 . Finally, from (2.1) the identity element of $\left(M_{h}, *\right)$ is $\mu_{0, h}=\mu_{0} h$ and the inverse of $\mu_{\alpha, h}$ is $\mu_{-\alpha, h}$. This completes the proof of Theorem 2.2.

By using Theorem 2.2, we immediately obtain a generalised Möbius inversion formula as follows. 
THEOREM 2.3. Let $h \in A(E)$ be any nonzero completely multiplicative function with $h(-1)=1$, and let $U, V$ denote complex-valued functions of a positive real variable t. Then

$$
V(t)=\sum_{k \in E_{;}|k| \leqslant t} \mu_{z}(k) U(t /|k|) h(k)
$$

for all $t>0$, if and only if

$$
U(t)=\sum_{k \in E ;|k| \leqslant t} \mu_{-z}(k) V(t /|k|) h(k)
$$

for all $t>0$.

Proof: (2.2) and (2.3) are equivalent to the statement

$$
V=U * \mu_{z, h} \Longleftrightarrow U=V * \mu_{-z, h},
$$

which follows from

$$
V=U * \mu_{z, h} \Longleftrightarrow V * \mu_{-z, h}=\left(U * \mu_{z, h}\right) * \mu_{-z, h}=U * \mu_{0, h}=U
$$

REMARK 1. Various consequences including some classical inversion formulas can be deduced from Theorem 2.3 by special choices of $E$, $z$, and $h(\cdot)$. In particular, the classical Möbius inversion pair is a particular case of (2.2) and (2.3) shown in Theorem 2.3 with $E=\mathbb{N}, z=1$, and $h(\cdot) \equiv 1$ (see [6] for a different approach).

Corollary 2.4 . Let $t_{0} \geqslant 1$, and let $h(k)$ be a nonzero completely multiplicative function. Then for all $t, 1 \leqslant t \leqslant t_{0}$, and complex-valued functions $F$ and $G$,

$$
G(t)=\sum_{1 \leqslant k \leqslant t} \mu_{z}(k) F(t / k) h(k)
$$

if and only if

$$
F(t)=\sum_{1 \leqslant k \leqslant t} \mu_{-z}(k) G(t / k) h(k)
$$

In Corollary 2.4, we may take

$$
\begin{aligned}
& F(t)= \begin{cases}f(t) & \text { if } t \in \mathbb{Z}, \\
0 & \text { if } t \notin \mathbb{Z},\end{cases} \\
& G(t)= \begin{cases}g(t) & \text { if } t \in \mathbb{Z}, \\
0 & \text { if } t \notin \mathbb{Z} .\end{cases}
\end{aligned}
$$


Hence, for $n \in \mathbb{N}$ we write (2.4) as

$$
\begin{aligned}
g(n)=G(n) & =\sum_{1 \leqslant k \leqslant n} \mu_{z}(k) F(n / k) h(k) \\
& =\sum_{k \mid n} \mu_{z}(k) f(n / k) h(k) \\
& =\sum_{d \mid n} \mu_{z}(n / d) f(d) h(n / d) .
\end{aligned}
$$

Similarly, from (2.5),

$$
\begin{aligned}
f(n)=F(n) & =\sum_{k \mid n} \mu_{-z}(k) g(n / k) h(k) \\
& =\sum_{d \mid n} \mu_{-z}(n / d) g(d) h(n / d) .
\end{aligned}
$$

Therefore, we obtain the following corollary.

Corollary 2.5. Let $h$ be any nonzero completely multiplicative function, and let $f$ and $g$ be complex-valued functions. Then for all $n \in \mathbb{N}$,

$$
g(n)=\sum_{k \mid n} \mu_{z}(k) f(n / k) h(k)
$$

if and only if

$$
f(n)=\sum_{k \mid n} \mu_{-z}(k) g(n / k) h(k)
$$

REMARK 2. Corollary 2.5 can be derived by using a similar argument to that in [1] with the consideration of the Abelian group $M_{h}=\left\{\mu_{z, h}=\mu_{z} h: z \in \mathbb{C}\right\}$ with respect to the addition * defined by

$$
\left(\mu_{\alpha, h} * \mu_{\beta, h}\right)(n):=\sum_{k \mid n} \mu_{\alpha}(k) h(k) \mu_{\beta}(n / k) h(n / k)=\mu_{\alpha+\beta, h}(n),
$$

where $h$ is a nonzero completely multiplicative function.

In Theorem 2.3, after substituting $F(t)=f(1 / t)$ and $G(t)=g(1 / t)$ into (2.2) and (2.3) and replacing $1 / t$ by $t$ afterwards, we can obtain the following results.

THEOREM 2.6. Let $h \in A(E)$ be any nonzero completely multiplicative function, and let $f$ and $g$ be complex-valued functions of positive real variable $t<1$,

$$
g(t)=\sum_{k \in E ;|k| \leqslant 1 / t} \mu_{-z}(k) f(|k| t) h(k)
$$


for all $0<t<1$, if and only if

$$
f(t)=\sum_{k \in E ;|k| \leqslant 1 / t} \mu_{z}(k) g(|k| t) h(k)
$$

for all $0<t<1$.

REMARK 3. (2.8) and (2.9) can be proved directly as follows.

Proof: If (2.8) holds, then

$$
\sum_{|k| \leqslant 1 / t} \mu_{z}(k) g(|k| t) h(k)=\sum_{|k| \leqslant 1 / t} \mu_{z}(k) h(k) \sum_{|m| \leqslant 1 /(|k| t)} \mu_{-z}(m) f(|m k| t) h(m) .
$$

By setting $m k=r$ on the right-hand side of the above equation and noting that $\mu_{z} * \mu_{-z}:=\sum_{m k=r} \mu_{z}(m) \mu_{-z}(k)=\mu_{0}(r)$, we can write it as follows.

$$
\begin{aligned}
\sum_{|m k| \leqslant 1 / t} \mu_{z}(k) \mu_{-z}(m) h(m k) f(|m k| t) & =\sum_{|r| \leqslant 1 / t} h(m k) f(|m k| t) \sum_{m k=r} \mu_{z}(k) \mu_{-z}(m) \\
& =h(1) f(t)=f(t) .
\end{aligned}
$$

Similarly, from (2.9) we can derive (2.8).

If we choose $E=\mathbb{N}$, then we have

COROLlary 2.7. Let $0<n_{0} \leqslant n_{1}$, and let $h(k)$ be any nonzero completely multiplicative function. For every $n_{0} \leqslant t \leqslant n_{1}$ and complex-valued functions $f$ and $g$,

$$
g(t)=\sum_{1 \leqslant k \leqslant n_{1} / t} \mu_{-z}(k) f(k t) h(k)
$$

if and only if

$$
f(t)=\sum_{1 \leqslant k \leqslant n_{1} / t} \mu_{z}(k) g(k t) h(k) .
$$

REMARK 4. A special case of $h \equiv 1$ and $z=1$ can be found in Theorems 268 and 270 of [5, Section 16.5].

\section{A Möbius Inversion formula for the Setting of the Selberg Multiplicative FunCtions}

We now give an application of Theorem 2.2 to the setting of Selberg-multiplicative functions. Similar to Theorems 2.1 and 2.2 , for a nonzero completely multiplicative function $h$ with $h(0)=1, A(E)_{h}:=\{f h: f \in A(E)-\{0\}\}$ forms an Abelian group with respect to the Dirichlet convolution defined in Definition 1.1. We shall show that $\left(\mathcal{G}_{h}, *\right)$ is a subgroup of $\left(A(E)_{h}, *\right)$. 
Let $F, G \in \mathcal{G}$; that is, for any $n \in \mathbb{N}, F(n)=\Pi_{p} f_{p}\left(e_{p}(n)\right)$ and $G(n)=\Pi_{p} g_{p}\left(e_{p}(n)\right)$, where for each prime $p f_{p}, g_{p}: \mathbb{N}_{0}=\mathbb{N} \cup\{0\} \mapsto \mathbb{C}$ with $f_{p}(0)=g_{p}(0)=1$. For each prime $p$ and a fixed nonzero completely multiplicative function $h_{p}$ with $h_{p}(0)=1$, we define

$$
q_{p}(r):=h_{p}(r) \sum_{\rho=0}^{r} f_{p}(\rho) g_{p}(r-\rho)\left(r \in \mathbb{N}_{0}\right)
$$

Thus $q_{p}(0)=1$. Furthermore, since $Q:=(F * G)_{h} \in A(E)_{h}$, where $h(n)=\Pi_{p} h_{p}\left(e_{p}(n)\right)$ and $h_{p}\left(e_{p}(n)\right):=h\left(p^{e_{p}(n)}\right)$, we have

$$
\begin{aligned}
Q(n) & =\Pi_{p} Q\left(p^{e_{p}(n)}\right)=\Pi_{p} \sum_{\rho=0}^{e_{p}(n)} F\left(p^{\rho}\right) G\left(p^{e_{p}(n)-\rho}\right) h\left(p^{e_{p}(n)}\right) \\
& =\Pi_{p} \sum_{\rho=0}^{e_{p}(n)} f_{p}(\rho) g_{p}\left(e_{p}(n)-\rho\right) h_{p}\left(e_{p}(n)\right)=\Pi_{p} q_{p}\left(e_{p}(n)\right),
\end{aligned}
$$

where the last two equalities are derived from the expressions of $F, G$, and $H$ and (3.1). Consequently, $(F * G)_{h} \in \mathcal{G}$ if $F h, G h \in \mathcal{G}_{h}$.

To prove that $F h \in \mathcal{G}_{h}$ implies its inverse $F^{-1} h \in \mathcal{G}_{h}$, for each prime $p$ we define

$$
g_{p}(0)=1, \sum_{\rho=0}^{r} f_{p}(\rho) g_{p}(r-\rho)=0
$$

for $r=1,2, \cdots$. Since $f_{p}(0)=1$ this is uniquely possible. From $\left(F * F^{-1}\right)_{h}=\varepsilon$, the identity of $\left(A(E)_{h}, *\right)$, we have

$$
0=\varepsilon\left(p^{r}\right)=h\left(p^{r}\right) \sum_{\rho=0}^{r} F\left(p^{\rho}\right) F^{-1}\left(p^{r-\rho}\right)=h\left(p^{r}\right) \sum_{\rho=0}^{r} f(\rho) F^{-1}\left(p^{r-\rho}\right)
$$

for each prime $p$ and $r \in \mathbb{N}$. Then from (3.2), we immediately obtain $F^{-1}(1)=1=g_{p}(0)$ and $F^{-1}\left(p^{r}\right)=g_{p}(r)$ for each prime $p$ and $r \in \mathbb{N}$. Consequently,

$$
F^{-1}(n)=\Pi_{p} F^{-1}\left(p^{e_{p}(n)}\right)=\Pi_{p} g_{p}\left(e_{p}(n)\right),
$$

where $F^{-1} \in \mathcal{G}$. Hence, constructed $F^{-1} h$ is the inverse of $F h$ in $\mathcal{G}_{h}$. (3.2) also give an algorithm to find $F^{-1}$.

We now use Lemmas 1.4 and 1.5 to derive the Möbius inversion formulas over $\left(\mathcal{G}_{h}, *\right)$ as follows.

THEOREM 3.1. Let either sequence of $\{\alpha(n)\}$ and $\{\beta(n)\}$ be given arbitrarily. For $F \in \mathcal{G}$, its inverse $G \in\left(\mathcal{G}_{h}, *\right)$ exists so that the general type of Möbius inversion formulas

$$
\beta(n)=\sum_{d \mid n} F(d) \alpha\left(\frac{n}{d}\right) h(d) \Longleftrightarrow \alpha(n)=\sum_{d \mid n} G(d) \beta\left(\frac{n}{d}\right) h(d)
$$

hold for any nonzero completely multiplicative function $h$ with $h(0)=1$ and for $\alpha, \beta$ : $\mathbb{N} \mapsto \mathbb{C}$. 
Proof: For given $F \in \mathcal{G}, F(n)=\Pi_{p} f_{p}\left(e_{p}(n)\right)$, from Lemma 1.4 we have

$$
\widehat{f}_{h}(s):=\sum_{n=1}^{\infty} F(n) h(n) n^{-s}=\Pi_{p} \sum_{p}^{\infty} f_{p}\left(e_{p}(n)\right) h_{p}\left(e_{p}(n)\right),
$$

where $\widehat{f}_{h}(s)$ is the Dirichlet formal series of the sequence $\{F(n) h(n)\}$ (see Definition 1.3). Solving system (3.2) or its equivalent form

$$
\left(\sum_{r=1}^{\infty} f_{p}(r) h_{p}(r) x^{r}\right)^{-1}=\sum_{r=1}^{\infty} g_{p}(r) h_{p}(r) x^{r}
$$

for each prime $p$ and $r=1,2, \cdots$. From (3.3), the inverse of $F(n) h(n)$ in $\left(\mathcal{G}_{h}, *\right)$ can be written as $G(n) h(n)$, where

$$
G(n)=\Pi_{p} g_{p}\left(e_{p}(n)\right)\left(g_{p}(0)=1\right) .
$$

Denote by $\widehat{g}_{h}(s)$ the Dirichlet formal series of the sequence $\{G(n) h(n)\}$. From Lemma 1.4, we obtain

$$
\widehat{g}_{h}(s):=\sum_{n=1}^{\infty} G(n) h(n) n^{-s}=\Pi_{p} \sum_{p}^{\infty} g_{p}\left(e_{p}(n)\right) h_{p}\left(e_{p}(n)\right) .
$$

Since (3.6) implies

$$
\widehat{g}_{h}(s)=1 / \widehat{f}_{h}(s),
$$

for the Dirichlet formal series, $\widehat{\alpha}(s): \mathbb{N} \mapsto \mathbb{C}$, of any given sequence $\{\alpha(n)\}$ we have $\widehat{\beta}(s):=\widehat{f}_{h}(s) \widehat{\alpha}(s)$ implies $\widehat{\alpha}(s)=\widehat{g}_{h}(s) \widehat{\beta}(s)$, and the last expression implies the previous one if $\widehat{\beta}(s)$ is indicated, namely

$$
\widehat{\beta}(s)=\widehat{f}_{h}(s) \widehat{\alpha}(s) \Longleftrightarrow \widehat{\alpha}(s)=\widehat{g}_{h}(s) \widehat{\beta}(s) .
$$

Obviously, from Lemma 1.5, inversion relation (3.10) is equivalent to (3.4). This completes the proof of the theorem.

REMARK 5. A special case of (3.4) for $h \equiv 1$ and a subgroup of $\mathcal{G} \equiv \mathcal{G}_{1}$ can be found in [2].

\section{REFERENCES}

[1] T.C. Brown, L.C. Hsu, J. Wang and P.J.S. Shiue, 'On a certain kind of generalized number-theoretical Möbius function', Math. Sci. 25 (2000), 72-77.

[2] P. Bundschuh, L.C. Hsu and P.J.S. Shiue, 'Generalized Möbius inversion- theoretical and computational aspects', (manuscript) (2003).

[3] N.-X. Chen, 'Modified Möbius inversion formula and its application to physics', Phys. Rev. Lett. 64 (1990), 1193-1195. 
[4] A. Fleck, 'Über gewisse allgemeine zahlentheoretische Funktionen, insbesondere eine der Funktion $\mu(n)$ verwandte Funktion $\mu_{k}(m)$ ', S.-B. Berlin. Math. Ges 15 (1916), 3-8.

[5] G.H. Hardy and E.M. Wright, An Introduction to the Theory of Numbers, (fifth edition) (Oxford University Press, New York, 1979).

[6] J. Knopfmacher, Abstract analytic number theory (North-Holland Publishing Co., Amsterdam, 1975).

[7] J. Sándor and A. Bege, 'The Möbius function: generalizations and extensions', Adv. Stud. Contemp. Math. (Kyungshang) 6 (2003), 77-128.

[8] A. Selberg, 'Remarks on multiplicative functions in: Number theory day', (Proc. Conf., Rockfeller Univ., New York), Lecture Notes in Mathematics 626 (Springer-Verlag, Berlin), pp. 232-241.

[9] H.S. Wilf, Generatingfunctionology (Academic Press, New York, 1990).

Department of Mathematics and Computer Science

nlinois Wesleyan University

Bloomington, IL 61702-2900

United States of America

Department of Mathematical Sciences

University of Nevada, Las Vegas

Las Vegas, NV 89154-4020

United States of America
Department of Mathematics

Dalian University of Technology

Dalian 116024

People's Republic of China 